\title{
Ae and A type shell stars
}

\section{Jaschek ${ }^{1}$ and Y. Andrillat ${ }^{2}$}

1 Observatoire de Strasbourg, URA 1280, CNRS, 11 rue de l'Université, 67000 Strasbourg, France

2 Laboratoire d'Astronomie, Université de Montpellier 2, URA 1280 CNRS, place Eugène Bataillon, 34095 Montpellier, Cedex 5, France

Received November 13, 1997; accepted January 26, 1998

\begin{abstract}
We present and discuss the observations of 14 $\mathrm{Ae}$ and $\mathrm{A}$ type shell stars in the visual, the $\mathrm{H} \alpha$ region and the near infrared. At least $57 \%$ of these stars are spectrum variables. The Paschen lines are formed in a region which has the characterics of giant stars. We find that the systems lie one magnitude above the main sequence and that a large percentage belong to double and triple systems.
\end{abstract}

Key words: stars: early type; emission line; variables

\section{Introduction}

The Ae and A type shell stars which constitute a small group with less than one hundred members, seems to be an extension of the well studied Be stars into the domain of lower temperatures. These stars have received less attention than the hotter Be type stars, as their variations are less spectacular than the changes in Be type stars and might be slower. Difficulties for describing the variations come from that most of the descriptions are only verbal descriptions, without recourse to measures (equivalent widths), which makes studies of variability rather uncertain.

A-type shell stars are of great interest since shell characteristics appear in three types of A-type objects, namely stars with Infrared (IRAS)excess (Jaschek et al. 1991), $\lambda$ Boo stars (Andrillat et al. 1995) and $\beta$ Pic objects. A better knowledge of the known shell stars should thus enable astronomers to improve the status of the frontier cases and to better delimit object categories. For example are shell systems an intermediate step toward $\beta$ Pic type systems?

The present paper provides some quantitative data for the red and infrared regions. We previously studied these

Send offprint requests to: Y. Andrillat 27 ter r.Adam de Craponne, 34000 Montpellier, France.
Table 1. Observational data

\begin{tabular}{cccccl}
\hline HD & HR & $V$ & blue & H3 & Paschen \\
\hline 77190 & 3589 & 6.07 & 11 & 14 & 15 \\
81728 & 3744 & 4.54 & 12 & & 15 \\
88195 & 3989 & 5.91 & 11 & 14 & 15 \\
98058 & 4368 & 4.47 & 11 & 13 & 12 \\
103287 & 4554 & 2.44 & 10 & 13 & 12 \\
108283 & 4733 & 4.95 & 10 & 13 & 12 \\
112028 & 4893 & 5.28 & 11 & 13 & 12 \\
118232 & 5112 & 4.70 & 12 & 13 & 12,10 \\
138629 & 5774 & 5.02 & 13 & 13 & 12,10 \\
148283 & 6123 & 5.52 & 10 & 13 & 12 \\
151431 & 6232 & 6.10 & 11 & & 12,15 \\
158352 & 6507 & 5.44 & 11 & & 12,15 \\
164577 & 6723 & 4.45 & 11 & & 15 \\
192518 & 7731 & 5.18 & 11 & 13 & 15 \\
\hline
\end{tabular}

Numbers given in this table are the dates in April 1996. (11= April 11, 1996).

stars (Jaschek et al. 1988, 1991), in both the photographic and the near infrared regions.

\section{Material}

We observed fourteen bright Ae and A-type shell stars (Table 1) at the Observatoire de Haute Provence (OHP) with the $152 \mathrm{~cm}$ telescope, in April 1996,with the Aurelie spectrograph (see Gillet et al. 1994) with a Thomson 7832 double bar detector, with 2048 photodiodes $(750 \times$ $13 \mu$ ). The grating had 300 grooves $/ \mathrm{mm}$, blazed at $6000 \mathrm{~A}$. The original dispersion is $33 \mathrm{~A} / \mathrm{mm}$, with a resolution of 1.3 A. Calibrations were made with a tungsten lamp for the flat field, and in wavelength by means of a thorium - argon hollow cathode for the blue and the red and thoriumneon for the near infrared.

Our intention was to observe these stars in three wavelength regions: in the blue $(\lambda 4000-4800)$, the $\mathrm{H}$ alpha $(=\mathrm{H} 3)$ region $(\lambda 6200-7000)$ and the Paschen region ( $\lambda 8100-8900)$, but due to cloudy nights this coverage 
Table 2. Classifications of the MK standards observed

\begin{tabular}{llll}
\hline a) in the blue region & \multicolumn{2}{c}{ b) in the red region } \\
\hline A 0 III & A 2 V & A 0 III & A $0 ~ V$ \\
A 3 III & A 3 V & A 2 V & \\
A 5 III & A 5 V & A 5 III & A 5 V \\
A 7 III & A 7 V & A 7 V & \\
F 0 III & F 0 V & F 0 III & A 9 V \\
\hline
\end{tabular}

could not be achieved for all stars. The stars were not observed simultaneously in the different ranges, but in view of the slowness of the changes, time differences of at most five days are probably unimportant. Several MK standards were observed for each region (see Table 2). We did not observe standards for the Paschen region, because these have already been published in Andrillat et al. (1995).

We have measured the equivalent widths with the computer program IHAP of ESO. The precision of the equivalent widths is of the order of $\pm 10 \%$ for medium and strong lines and somewhat less for the weaker lines. The equivalent widths are given in Tables 3 and 4 for the red and infrared regions respectively. No values were measured in the blue region, due to the large number of metallic lines which produce many blends.

Table 3. Equivalent width of absorption lines in the $\mathrm{H}$ a region

\begin{tabular}{ccllllll}
\hline HD & H $\alpha$ & 6277 & 6346 & 6370 & 6433 & 6495 & 6516 \\
\hline & $?$ & Si II & Fe II & Fe II & Fe II & Fe II & \\
\hline 77190 & 10.4 & & $\mathrm{p}$ & $\mathrm{p}$ & & $\mathrm{p}$ & $\mathrm{p}$ \\
88195 & $8.7^{*}$ & & $\mathrm{p}$ & $\mathrm{p}$ & & & $\mathrm{p}$ \\
98058 & 9.5 & & & & & & \\
103287 & 11.6 & & $\mathrm{p}$ & & & $\mathrm{p}$ & $\mathrm{p}$ \\
108283 & 8.1 & $\mathrm{p}$ & $\mathrm{p}$ & $\mathrm{p}$ & $\mathrm{p}$ & $\mathrm{p}$ & $\mathrm{p}$ \\
112028 & $8.8^{*}$ & $\mathrm{p}$ & $\mathrm{p}$ & $\mathrm{p}$ & $\mathrm{p}$ & $\mathrm{p}$ & $\mathrm{p}$ \\
118232 & 11.5 & $\mathrm{p}$ & $\mathrm{p}$ & $\mathrm{p}$ & $\mathrm{p}$ & $\mathrm{p}$ & $\mathrm{p}$ \\
138629 & 10.5 & $\mathrm{p}$ & $\mathrm{p}$ & $\mathrm{p}$ & & & $\mathrm{p}$ \\
148283 & 9.8 & $\mathrm{p}$ & $\mathrm{p}$ & $\mathrm{p}$ & & & $\mathrm{p}$ \\
192518 & $10.0^{*}$ & $\mathrm{p}$ & $\mathrm{p}$ & $\mathrm{p}$ & $\mathrm{p}$ & & $\mathrm{p}$ \\
\hline
\end{tabular}

* A sharp absorption core exists, superimposed upon the broad absorption line.

Equivalent widths given in A.

p means visible, but not measured.

The lines $\lambda \lambda$ 6347(Si II M.2), 6371(Si II M.2), 6456(Fe II M.74), 6496(Ba II M.2) and 6516(Fe II M.40) are absent in A0 V (except 6516) appear in A2 and strenghten with advancing type toward late A. No difference in the behavior of these lines seem to exist between the class III and $\mathrm{V}$ stars in this spectral range.

\section{Individual objects}

We provide next a short description of the spectra.
Table 4. Equivalent width of absorption lines measured in the Paschen region

\begin{tabular}{lclccllc}
\hline HD & P12 & P13* & P14 & P15* & P16* & P17 & OI \\
\hline 77190 & 4.4 & 4.3 & 1.7 & 2.6 & 1.4 & 0.28 & 0.53 \\
81728 & 7.5 & 6.6 & 3.7 & 3.0 & 1.8 & 0.65 & 0.84 \\
88195 & 7.2 & 6.7 & 4.6 & 3.8 & 2.6 & 1.4 & 1.48 \\
98059 & 5.1 & 6.1 & 2.9 & 3.9 & 2.4 & 0.78 & 0.80 \\
103287 & 7.8 & 5.8 & 3.6 & 2.5 & 1.4 & 0.40 & 0.68 \\
108283 & 4.2 & 5.9 & 2.5 & 4.4 & 2.8 & 0.81 & 0.81 \\
112028 & 7.1 & 6.8 & 4.2 & 3.9 & 2.4 & 1.1 & 1.45 \\
118232 & 6.4 & 5.6 & 3.0 & 2.9 & 1.8 & 0.66 & 0.65 \\
id & 6.5 & 5.4 & 2.8 & 2.8 & 1.7 & 0.60 & 0.70 \\
138629 & 7.1 & 6.2 & 3.9 & 3.5 & 2.25 & 1.05 & 0.72 \\
id & 7.2 & 6.3 & 3.8 & 3.4 & 2.1 & 0.99 & 0.81 \\
148283 & 5.4 & 5.6 & 2.7 & 3.3 & 2.0 & 0.49 & 0.80 \\
151431 & 6.8 & 6.1 & 3.6 & 3.0 & 2.0 & 1.1 & 0.73 \\
id & 6.9 & 5.9 & 3.8 & 2.8 & 1.75 & 0.57 & 0.52 \\
158352 & 4.7 & 4.55 & 2.3 & 3.3 & 1.65 & 0.51 & 0.55 \\
id & 4.7 & 4.7 & 2.1 & 2.9 & 1.4 & 0.39 & 0.42 \\
164577 & 7.7 & 5.7 & 3.7 & 2.2 & 1.2 & 0.77 & 0.56 \\
192518 & 5.7 & 6.3 & 3.2 & 4.1 & 2.5 & 0.92 & 0.97 \\
\hline
\end{tabular}

Note $^{*}$ blend with Ca II.

OI is blended with $\mathrm{P} 18$.

Equivalent widths given in A.

HD 77190. We see no shell features neither in the blue, nor in the red. The Paschen region shows a normal $R$ ratio (defined as $\mathrm{P} 17 / \mathrm{P} 12$, see below) and the $\mathrm{P} 15 / \mathrm{P} 14$ ratio is consistent with the blue spectral type $\mathrm{A} 8 \mathrm{~V}$. The only anomaly is that the $\mathrm{O}$ I line is too strong for the type.

HD 81728. In the blue we see absorption cores in H4, 5 and 6 . Fe II lines from M.27 and 38 are enhanced, as well as Ti II, M.41 and 82. In the Paschen region the $R$ ratio is normal, but $W(\mathrm{P} 14)$ is too strong for the spectral type A $2 \mathrm{~V}$. Ca lines are strong and narrow, which is in contradiction with the type, since at A2 no Ca II should be visible. O I is strong and narrow. A shell is thus certainly present.

HD 88195. In the blue, Fe II lines (M.27, 38) and Ti II (M.41, 82) are enhanced. In the red, H 3 has a strong and narrow superposed central absorption core. In the red, Fe II and Si II are too strong for the type. In the Paschen region, $W(\mathrm{P} 14)$ is too strong for type $\mathrm{V}$ and the Paschen ratio corresponds to LC (=luminosity class) III. $\mathrm{O}$ I is very strong and Ca II probably in emission, since $W(\mathrm{P} 15)<W(\mathrm{P} 14)$. A shell is thus certainly present.

HD 98058. In the blue one sees no Balmer line cores, but Fe II (M.38) and Ti II (M.41 and 82) lines are enhanced. In the red, $\mathrm{H} 3$ is normal. In the Paschen region the $W(\mathrm{P} 14)$ and the ratio $W(\mathrm{P} 14) / W(15)$ are consistent with the blue spectral type. However the $R$ ratio corresponds to LC III and O I is too strong for the type. A weak shell 
Table 5. Equivalent width of the absorption lines measured in the Paschen region, in 1989

\begin{tabular}{cccccccl}
\hline HD & & P13* & P14 & P15* & P16* & P17 & OI \\
\hline 81728 & 21.4 & 6.2 & 3.8 & 3.4 & 1.7 & & \\
& 22.4 & 6.5 & 3.6 & 3.2 & 1.8 & 0.58 & 0.55 \\
& 23.4 & 6.7 & 4.1 & 3.5 & 2.1 & 0.85 & 0.55 \\
88195 & 19.4 & 7.0 & 4.6 & 3.9 & 2.8 & 1.5 & 1.1 \\
& 19.4 & 6.5 & 4.8 & 3.9 & 2.7 & 1.2 & 0.90 \\
98058 & 18.4 & 5.8 & 3.0 & 4.2 & 2.5 & 0.88 & $0.45:$ \\
& 18.4 & 5.7 & 3.1 & 4.1 & 2.6 & 0.88 & $0.65:$ \\
103287 & $07.2^{*}$ & 6.1 & 3.9 & 2.7 & 1.5 & 0.63 & 0.46 \\
108283 & 18.4 & 5.8 & 2.8 & 4.7 & 3.1 & 1.1 & \\
& 18.4 & 5.7 & 2.8 & 4.5 & 2.9 & 0.95 & \\
112028 & 15.6 & 7.1 & 4.4 & 3.9 & 2.4 & 1.1 & $1.37:$ \\
& 15.6 & 7.0 & 4.4 & 3.9 & 2.3 & 1.1 & $1.33:$ \\
118232 & 19.4 & 5.8 & 3.1 & 3.1 & 1.7 & 0.73 & $0.71:$ \\
& 19.4 & 5.7 & 2.8 & 2.9 & 1.7 & 0.65 & $0.55:$ \\
138629 & 19.4 & 5.6 & 2.9 & 3.0 & 1.8 & 0.60 & $0.65:$ \\
148283 & 19.4 & 6.5 & 4.2 & 3.6 & 2.3 & 1.3 & $0.62:$ \\
158352 & 19.4 & 5.4 & 3.0 & 3.5 & 2.0 & 0.91 & \\
164577 & 19.4 & 5.6 & 4.1 & 2.7 & 1.4 & 0.91 & $0.33:$ \\
& 19.4 & 5.5 & 3.9 & 2.6 & 1.4 & 0.80 & $0.26:$ \\
192518 & 21.4 & 5.4 & 3.3 & 4.4 & 2.6 & 0.95 & $0.73:$ \\
\hline
\end{tabular}

Second column, date (day, month) of 1989. * Stands for 1988. Equivalent widths given in A.

is thus present.

HD 103287. In the red H 3 seems normal, as well as the metallic lines. In the Paschen region the $W(\mathrm{P} 14)$ and the $W(\mathrm{P} 14) / W(\mathrm{P} 15)$ ratio are both consistent with the A $0 \mathrm{~V}$ spectral type. The $R$ ratio is normal and $\mathrm{O} \mathrm{I}$ is also practically normal.

HD 108283. In the blue we observe a rich shell spectrum with enhanced lines of Sc II (M.7), Ti II (M.40, 41, 82), Cr II (M.30), Fe II (M.2, 28,37, 38, 42) and Sr II (M.1). In the Paschen region, $W(\mathrm{P} 14)$ is too strong and the $R$ ratio corresponds to LC III. Ca II lines are narrow and OI is strong for the type, although weaker than in 1991. A shell is thus present.

HD 112028. In the red one observes a clear central absorption core in H 3 and the metallic lines are enhanced. In the Paschen region, the ratio $R$ corresponds to LC III, $\mathrm{Ca}$ II lines are narrow, a fact which disagrees with the blue spectral type A 1 . Furthermore the profiles of P13, 15 and 16 are deformed on the red border, which implies probably a structure unresolved at our dispersion. O I is very strong. The Paschen spectrum agrees with the 1991 description. A shell is present.

HD 118232. In the red, $H 3$ has a normal profile, but $W(\mathrm{H} 4)$ is too large. In the Paschen region, the $R$ ratio is normal, but $W(\mathrm{P} 14)$ is too large and the ratio $W(\mathrm{P} 15) / W(\mathrm{P} 14)$ is abnormal, which is probably due to Ca II in emission, since $W(\mathrm{P} 14)>W(\mathrm{P} 15)$. O I is very strong, although smaller than in 1991. A shell is present.

HD 138629. In the red, $\mathrm{H} 3$ has a normal profile, but $W(\mathrm{H}$ 4 ) is too large. The $R$ ratio is normal and the strength of $\mathrm{P} 14$ and the $W(\mathrm{P} 14) / W(\mathrm{P} 15)$ ratio are both compatible with the spectral type. O I is also about normal for the type. No clear evidence of a shell.

HD 148283. In the blue, the hydrogen lines have normal profiles and there are only indications of a few weak shell lines of Fe II $(\mathrm{M} .27,38)$ and Ti II (M.41, 82). In the red $\mathrm{H} 3$ also has a normal profile and the metallic lines are weak for the type. In the Paschen region the $R$ ratio is normal and the strength of $\mathrm{P} 14$ and the ratio $W(\mathrm{P} 14) / W(\mathrm{P} 15)$ are normal for the type. O I is normal or weak for the type. Weak shell present.

HD 151431. In the blue, the Balmer lines have normal profiles and only a few Ti II lines are somewhat stronger than expected. In the Paschen region the $R$ ratio corresponds to LC III and both $W(\mathrm{P} 14)$ and the ratio $W(\mathrm{P} 14) / W(\mathrm{P} 15)$ are abnormal. O I is too strong for the type. A shell is present.

HD 158352. In the blue, there are no clear indications of a shell. The $R$ ratio, $W(\mathrm{P} 14)$ as well as the $W(\mathrm{P} 14) / W(\mathrm{P} 15)$ ratio are normal for the type. Also O I is about normal, in contrast to 1991 when it was definitely stronger. Apparently the shell has weakened or disappeared.

HD 164577. In the blue, no clear traces of a shell are seen. In the Paschen region, $W(\mathrm{P} 14)$ is strong for the type and the $R$ ratio corresponds to LC III. O I is normal. A shell is present.

HD 192518. In the blue one observes strong Fe II lines from M.37 and 38. In the Paschen region, $W(\mathrm{P} 14)$ is too strong for the type and the $R$ ratio corresponds to LC III. O I is strong, but has not changed from 1991. Evidence of a shell.

\section{Variability}

We discuss the variability of these objects. As descriptions made before 1988 have been summarized by us (Jaschek et al. 1988) for the blue region, we include thus mostly descriptions which appeared after 1988 for the blue. Similarly we include only descriptions made after 1986 for the $\mathrm{H}$ alpha region (Andrillat et al. 1986) and after 1991 for the Paschen region (Jaschek et al. 1991). The observations reported in our 1991 paper were made 
Table 6. Binarity of our sample

\begin{tabular}{ll}
\hline HD & Notes \\
\hline 77190 & double, $\Delta m=2.8 s=103^{\prime \prime}$ \\
81728 & triple system: A, B $\Delta m=0, s=0^{\prime \prime} 3 \mathrm{C}$ at $10^{\prime \prime} 7$ \\
88195 & binary (speckle) $0^{\prime \prime} 2(1)$ \\
98058 & double $\Delta m=4.7, s=96^{\prime \prime}$ \\
103287 & examined by speckle with negative result $(1)^{*}$ \\
108283 & $*$ \\
112028 & triple system. B, C at $2^{\prime \prime} 2$ \\
118232 & examined by speckle with negative result $(1)$ \\
138629 & speckle binary $0^{\prime \prime} 06(2)$ \\
148283 & speckle binary $0^{\prime \prime} 2(3)$ \\
151431 & double $\Delta m=3.6 s=23^{\prime \prime}$ \\
158352 & Examined by speckle with negative result $(3)$ \\
164577 & double $\Delta m=2.5 s=0^{\prime \prime} 6^{*}$ \\
192518 & examined by speckle with negative result $(3)$ \\
\hline
\end{tabular}

Indications of binarity from Hoffleit \& Jaschek (1982). Speckle observations code is given below.

* noted as SB in Bright Stars fourth Hoffleit D. and Jaschek C. (eds.) 1982, Yale Univ. Observ.

(1) McAlister H.A, Mason B.D., Hartkopf W.I., Shara M.M., 1993, AJ 106, 1639

(2) Hartkopf W.I., McAlister H.A., Franz O.G., 1989, AJ 98, 1014

(3) McAlister H.A., Hartkopf W.I., Hutter D.J., Shara M.M., Franz O.G., 1987, AJ 92, 183.

in 1989 , so that when discussing variability, we compare the years of observations, not those of the publication of the paper.

To better use the descriptions of the infrared spectra taken in 1989, these have been measured. The measures, as far as they concern stars observed in this paper, are listed in Table 5. This should replace advantageously the former verbal descriptions.

HD 77190. A shell was present in 1973; in 1983 at $\mathrm{H} \alpha$ and now no traces of it are seen.

HD 81728. In 1977 Andersen \& Nordstroem called the object a "possible shell star"; in 1984 H3 had a sharp absorption core upon a broad photospheric line; in 1988 the shell was definitely present and is weakly present now. This agrees with the $\mathrm{O}$ I line which is weaker now than in 1989. The Ca II profiles are also broader.

HD 88195. The shell was not noticed by Cowley et al. (1969), neither by Dworetsky (1974) It was detected by Andersen \& Nordstroem (1977) and was present in 1984 in the $\mathrm{H} 3$ region under the form of large photospheric absorption line, weak central emission and strong central reversal. The shell was present in a weakened form in 1988. It continues to be present and O I 8446 is weaker now than in 1988.
Table 7. Absolute magnitudes of the stars of our sample

\begin{tabular}{cccc}
\hline HD & $M$ (vis) & $M$ (corr) & $B-V$ \\
\hline 77190 & $2.23 \pm 0.11$ & 2.24 & 0.24 \\
98058 & $0.56 \pm 0.11$ & & 0.21 \\
103287 & $0.36 \pm 0.05$ & 0.56 & 0.05 \\
108283 & $0.30 \pm 0.13$ & 0.50 & 0.28 \\
118232 & $0.85 \pm 0.09$ & 1.05 & 0.13 \\
138629 & $-0.58 \pm 0.15$ & 0.17 & 0.07 \\
148283 & $1.05 \pm 0.08$ & 1.80 & 0.17 \\
158352 & 1.41 & & 0.24 \\
164577 & $-0.13 \pm$ & -0.02 & 0.05 \\
192518 & 0.18 & & 0.19 \\
\hline
\end{tabular}

$M$ (corr) are corrected for binarity.

HD 98058. With some variations in intensity, the shell is present since 1973 at least. However in 1984 the H3 line was normal. Comparing equivalent widths of 1988 and 1996, Paschen lines have remained constant, Ca II has strengthened and probably also O I.

HD 103287. The emission phenomena in this star seem to concentrate in the Ca II and H3 lines. O I also varied, passing from 0.46 in 1989 to 0.68 in 1996. Notice that on 1983 and 1984 the H3 line was strongly variable both in equivalent width and in structure; on some plates a weak central emission was present.

HD 108283. (=14 Com). This is a star with a rather stable shell. We observed no perceptible changes in the Paschen region. In 1983-4 the H3 profile was normal, without structure.

HD 112028. This is also a rather stable shell, with no changes in the Paschen region. In 1983-4 the H3 line had a strong absorption profile, with a central reversal and superposed central reversal and $V / R$ variations; in 1996 we find a sharp absorption core.

HD 118232. The shell was described by Abt \& Moyd (1973), disappeared afterwards (1977-1988) and seems to be back now. Comparing the Paschen region in 1989 and 1996, no noticeable changes are seen. Notice also that the H3 profile was normal in 1983-4 and in 1996, but at the latter date its equivalent width is too large for the type.

HD 138629. Apparently a weak shell, stable over the years. H3 has decreased from 11.6 in 1986 to 10.5 and the same is true for P 14 between 1989 and 1996. Notice also that the H3 line in 1983-4 showed sometimes asymmetric profiles, which conducted to a search of short term variability which was inconclusive. In 1996 the equivalent width of the line is too large for the spectral type.

HD 148283. The shell is rather weak but seems stable. O I seems to have diminished. No changes in the Paschen 


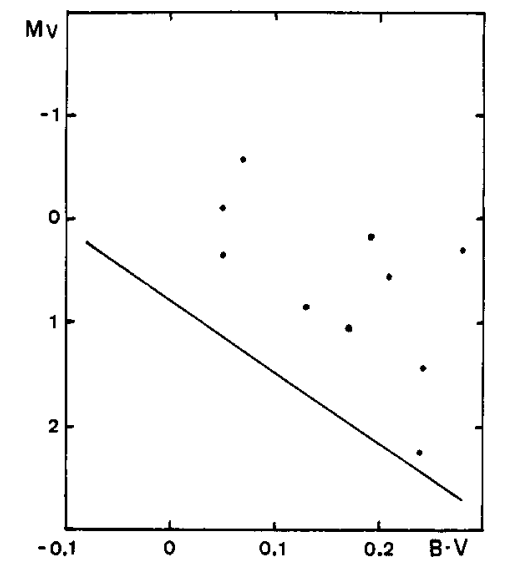

Fig. 1. The absolute magnitudes of the Ae and A type shell stars of our sample, based upon HIPARCOS parallaxes. The continuous line represents the ZAMS

region between 1989 and 1996 .

HD 151431. Apparently no variations between 1991 and our plates.

HD 158352. The shell was described by Abt \& Moyd (1973); Dominy \& Smith (1977) and Slettebak (1982). The latter author denoted a weakening of the features, which by 1988 had disappeared except for the cores in the blue Ca II lines. With respect to 1989 , we find a strengthening of the Ca II lines and of O I 8446.

HD 164577. No traces of a shell. The star had only a weak centrally reversed emission in H3. In 1983-4 the H3 line showed a structure: with central emission and central reversal and $V / R$ variations. In the Paschen region we find a strengthening of O I 8446 by a factor of almost two in 1996 with respect to 1988 .

HD 192518. Apparently a stable shell with some variations in strength over the years. In the Paschen region we find a strengthening of Ca II and O I 8446 from 1989 to 1996.

This discussion shows that we need considerably more observations to say something definitive about the variability of the group. We found strong variability (i.e. disappearance or appearance) in seven stars (HD 77190, $81728,88195,103287,118232,158352$ and 164577), stable shells (with some intensity variations) in six cases and one uncertain case. In the seven cases of variability, some time scales are of the order of decades - but since this corresponds more or less to the time interval between the different surveys this is less significant than one would like. However in at least one case there is evidence of changes on a short time scale, namely HD 138629 for which we found variations over a few days. We find thus at least eight stars out of fourteen to be variable, i.e. $57 \%$. However if we apply the variability count strictly to the sample of 1988 and

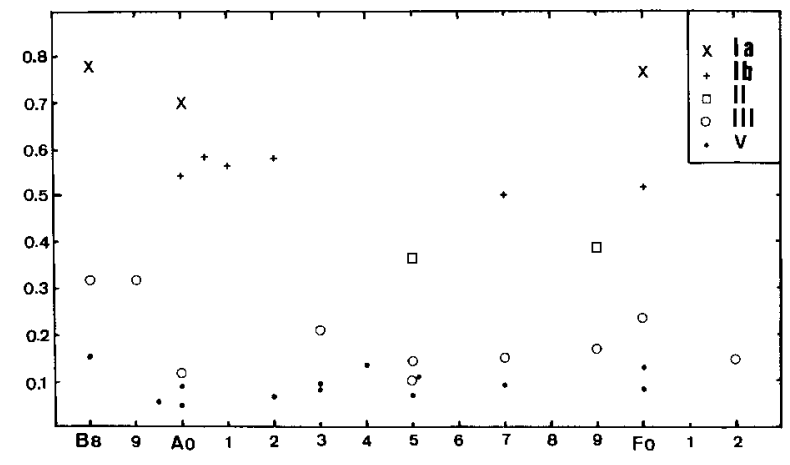

Fig. 2. The $R$ ratio as a function of spectral type and luminosity class for standards MK stars

1996, we find only three stars in which the situation has changed, i.e. $25 \%$. Thus a close following of the stars will reveal more variations, especially if the variations can be documented by measures of equivalent widths. In successive papers the percentage of stars found to be variable has increased, and is now practically two thirds of all objects.

\section{5. $R$ ratio}

We have defined an $R$ ratio to characterize the behavior of the Paschen series. We defined as such the ratio $W(\mathrm{P} 17) / W(\mathrm{P} 12)$, because we cannot use $\mathrm{P} 18$ because of its contamination with $\mathrm{O}$ I and $\mathrm{P} 11$ because it is too near to the border of the observed region to be trustworthy.

Figure 1 is a plot of the $R$ ratio in normal stars. The stars were taken from Andrillat et al. (1995). The ratios fall in three different regions for supergiants, giant and dwarfs, separated by the values 0.35 and 0.13 . From Table 5 one can derive the ratios for the shell stars of our sample and verify that in the case of well visible shells, the ratios correspond to luminosity class III rather than V, where the spectral classifications would put the star. Since the Paschen lines are produced somewhere in the shell, this implies simply that the physical conditions there resemble more the atmosphere of a giant than that of a dwarf. A theoretical paper discussing the $R$ ratio by Houziaux \& Fremat is forthcoming.

\section{Binarity}

We examined the literature for the binarity of our objects (Table 6). Out of 14 objects, at least nine belong to double or triple systems. For the remaining five we have negative speckle information for four and in one case there exists an indication that the star might be a spectroscopic binary. Such an indication is however doubtful, since often the sharp shell lines and the broader hydrogen lines reveal a difference of radial velocities, which is taken as an indication of binarity. In summary only one-third are single 
objects, or, if we refer to the totality of the components present, 5 out of 25 , i.e. $20 \%$ This proportion is lower than the one we found in our 1988 paper.

\section{Parallaxes}

Previously we could only suggest that the stars appeared to be giants, based upon lines of evidence coming from colors, photometric indices and color-spectral type relations. With the HIPPARCOS data we can now provide absolute magnitudes with errors of the absolute magnitudes is equal or less than \pm 0.3 . The corresponding values are quoted in Table 7 , where the absolute magnitudes were corrected for binarity, if necessary. Figure 2 shows that on the average the stars lie $1^{\mathrm{m}} 35 \pm 0.76$ above the main sequence. This is equivalent to a radius larger by a factor of $4.7 \pm 3$ implying that the average radius of a shell star is about 4.7 times as large as the normal radius of a dwarf star. The shell is thus located rather close to the star. In Be stars we found from a different procedure $3.2 \pm 1.4$ (Jaschek \& Jaschek 1992). Since within the errors the two values coincide, this underlines the close similarity of both kinds of objects.

\section{Summary}

We have described the spectra of 14 Ae and A type shell stars in three different spectral regions observed during one observing run at the OHP in 1996. We discuss the behavior over time of the 14 stars and conclude that at least $57 \%$ show spectrum variability. For several stars there exist now well documented changes from a normal phase to a shell phase.
We analysed the binarity of our sample and found that a large percentage of the objects belong to double or triple systems. Further stars with clear indications of a shell tend to have characteristics of the Paschen series similar to giants, rather than to the dwarf stars they really are. Such a behavior is confirmed by the overbrightness of the A shell stars which reaches $1^{\mathrm{m}} 35$ magnitude, implying that the emitting surface is larger by a factor of about three than that of a normal dwarf.

Acknowledgements. We thank Mr. J. Marcout from the Strasbourg Observatory for drawing Figs. 1 and 2. We thank also the referee, Dr. S. Adelman, for improving the manuscript.

\section{References}

Abt H.A., Moyd K.L., 1973, ApJ 182, 809

Andersen J., Nordstroem B., 1977, A\&AS 29, 309

Andrillat Y., Jaschek M., Jaschek C., 1986, A\&AS 65, 1

Andrillat Y., Jaschek C., Jaschek M., 1995, A\&AS 112, 475

Andrillat Y, Jaschek C., Jaschek M., 1995, A\&A 299, 43

Cowley A., Cowley C., Jaschek M., Jaschek C., AJ 74, 375

Dominy J.F., Smith M.A., 1977, ApJ 217, 494

Dworetsky M.M., 1974, ApJ S28, 101

Gillet D., Burnage R., Kohler D., et al., 1994, A\&A 108, 181

Jaschek M., Jaschek C., Andrillat Y., 1988, A\&AS 72, 505

Jaschek M., Andrillat Y., Jaschek C., 1991, A\&A 250, 127

Jaschek C., Jaschek M., Andrillat Y., Egret D., 1991, A\&A 252 229

Jaschek C., Jaschek M., 1992, A\&AS 95, 535

Jaschek C., 1997, Hipparcos Venice '97, ESA SP 402, p. 429

Slettebak A., 1982, ApJS 50, 55 